\title{
A study on some generalized multiplicative and generalized additive arithmetic functions
}

\author{
D. Bhattacharjee \\ Department of Mathematics, North-Eastern Hill University \\ Permanent Campus, Shillong-793022, India \\ e-mails:debashis_bhattacharjee@yahoo.com, \\ dbhattacharjee@nehu.ac.in
}

\begin{abstract}
In this paper by an arithmetic function we shall mean a real-valued function on the set of positive integers. We recall the definitions of some common arithmetic convolutions. We also recall the definitions of a multiplicative function, a generalized multiplicative function (or briefly a GM-function), an additive function and a generalized additive function (or briefly a $\boldsymbol{G A}$-function). We shall study in details some properties of $\boldsymbol{G} \boldsymbol{M}$-functions as well as $\boldsymbol{G A}$-functions using some particular arithmetic convolutions namely the Narkiewicz's $A$-product and the author's $B$-product. We conclude our discussion with some examples.
\end{abstract}

Keywords: Arithmetic function, Multiplicative function, Arithmetic convolution, Dirichlet convolution, Narkiewicz's $A$-product, $B$-product, Multiplicative $B$-product, $\boldsymbol{G A}$-function, GM-function.

2010 Mathematics Subject Classification:11A25.

\section{Introduction}

In this paper, by an arithmetic function we shall mean a real valued function on the set of positive integers. The early history of the theory of arithmetic functions is contained in the first volume of Dickson's [13] monumental "History of the Theory of Numbers". The convolution operation played a prominent role from the very beginning. Many results from early times involved the convolution of two or more particular arithmetical functions.

Early in the $19^{\text {th }}$ century the Dirichlet convolution, as well as addition and multiplication, began to be viewed as binary operations on the set of arithmetic functions. In the works of Cipolla [10] and Bell [2] it was recognized that the arithmetic functions form a commutative ring with unity with respect to addition and convolution. 
The study of the structure of the ring of arithmetic functions has continued and we point out papers of, Vaidyanathaswamy [25], Cohen [11], Narkiewicz [19], Subbarao [22], Scheid [20], Apostol [1], Davison [12], Gioia [14], McCarthy [18], Sivaramakrishnan [21], Haukkanen [16], Tóth [23, 24], Lehmer [17], Bhattacharjee [3-7], Bhattacharjee and Saikia [8], and others.

We now introduce some of the most common arithmetic convolutions to our readers.

1. Natural product (usual product). If $f$ and $g$ are arithmetic functions, we define the natural product $f g$ (also written as $f \times g$ ) as the arithmetic function whose value at any positive integer $n$ is given by:

$$
(f g)(n)=f(n) g(n)
$$

2. Dirichlet product (convolution). If $f$ and $g$ are arithmetic functions, we define the Dirichlet product (or Dirichlet convolution) $f * g$ as the arithmetic function whose value at any positive integer $n$ is given by:

$$
(f * g)(n)=\sum_{d \mid n} f(d) g\left(\frac{n}{d}\right)=\sum_{d_{1} d_{2}=n} f\left(d_{1}\right) g\left(d_{2}\right)
$$

3. Unitary product (convolution). If $f$ and $g$ are arithmetic functions, we define the unitary product (or unitary convolution) $f \circ g$ as the arithmetic function whose value at any positive integer $n$ is given by:

$$
(f \circ g)(n)=\sum_{\substack{d_{1} d_{2}=n \\\left(d_{1}, d_{2}\right)=1}} f\left(d_{1}\right) g\left(d_{2}\right)
$$

where $\left(d_{1}, d_{2}\right)$ stands for the greatest common divisor of $d_{1}$ and $d_{2}$.

4. L.C.M. product (convolution). If $f$ and $g$ are arithmetic functions, we define the L.C.M product (or L.C.M convolution) $f \oplus g$ as the arithmetic function whose value at any positive integer $n$ is given by

$$
(f \oplus g)(n)=\sum_{\left[d_{1}, d_{2}\right]=n} f\left(d_{1}\right) f\left(d_{2}\right)
$$

where $\left[d_{1}, d_{2}\right]$ stands for the least common multiple of $d_{1}$ and $d_{2}$.

5. The $k$-product (convolution). If $f$ and $g$ are arithmetic functions, we define the $k$-product $f *_{k} g$ as the arithmetic function whose value at any positive integer $n$ is given by:

$$
\left(f *_{k} g\right)(n)=\sum_{a b=n} f(a) g(b) k((a, b))
$$

where $k((a, b))$ is a function of $(a, b)$, the greatest common divisor of $a$ and $b$.

6. Davison's product (convolution). If $f$ and $g$ are arithmetic functions, we define the Davison's product $f *_{D} g$ as the arithmetic function whose value at any positive integer $n$ is given by:

$$
\left(f *_{D} g\right)(n)=\sum_{a b=n} f(a) g(b) A(a, b)
$$

where $A(a, b)$ is a function of two variables $a$ and $b$, instead of being a function of their greatest common divisor as in $\mathbf{5}$. 
7. The Lehmer $\boldsymbol{\psi}$-product (convolution). Let $\psi(x, y)$ be a positive integral-valued function defined for a prescribed set $T$ of ordered pairs $(x, y)$ such that $x, y \in \mathbb{N}$, where $\mathbb{N}$ denotes the set of all positive integers. Then the $\psi$-product $f \odot g$ of $f$ and $g$ is defined by:

$$
(f \odot g)(n)=\sum_{\psi(a, b)=n} f(a) g(b),
$$

where $n \in \mathbb{N}$.

8. Narkiewicz's $\boldsymbol{A}$-product (convolution). For every positive integer $n$ fix the set $A_{n}$ of some divisors of $n$. For arithmetic functions $f$ and $g$ their $A$-product $f *_{A} g$ is given by

$$
\left(f *_{A} g\right)(n)=\sum_{d \in A_{n}} f(d) g\left(\frac{n}{d}\right)
$$

for $n=1,2,3, \ldots$.

We now recall the definitions of a multiplicative function, a generalized multiplicative function (or briefly a $\boldsymbol{G M}$-function), an additive function and a generalized additive function (or briefly a $\boldsymbol{G A}$-function). We shall study in details some properties of $\boldsymbol{G M}$-functions as well as $\boldsymbol{G A}$-functions using some particular arithmetic convolutions namely the Narkiewicz's $A$-product and the author's $B$-product as multiplicative binary operations.

An arithmetic function $F$ is said to be multiplicative if $F(1)=1$ and $F(m n)=F(m) F(n)$, whenever $(m, n)=1$, where $(m, n)$ stands for the greatest common divisor of $m$ and $n$.

There are several generalizations of multiplicative functions which can be found in McCarthy [18], Sivaramkrishnan [21]. Zafrullah in his paper [26] introduced a new generalization where he defined an arithmetic function $F$ to be a Generalized Multiplicative function (or briefly a $\boldsymbol{G M}$-function) if $F(1)=1$ and there exists a multiplicative function $f$ such that:

$$
F(m n)=F(m)^{f(n)} F(n)^{f(m)}
$$

whenever $(m, n)=1$.

An arithmetic function $F$ is said to be an additive function if $F(m n)=F(m)+F(n)$ whenever $(m, n)=1$. We call an arithmetic function $F$ to be a Generalized Additive function (or briefly a $\boldsymbol{G A}$-function) if there exists a multiplicative function $f$ such that

$$
F(m n)=F(m) f(n)+F(n) f(m)
$$

whenever $(m, n)=1$.

Haukkanen in his paper [15] studied some properties of $\boldsymbol{G} \boldsymbol{M}$-functions and $\boldsymbol{G A}$-functions considering Dirichlet convolution as the multiplicative binary operation. The present author in this paper tries to study some properties of $\boldsymbol{G} \boldsymbol{M}$-functions and $\boldsymbol{G} \boldsymbol{A}$-functions by considering the Narkiewicz's $A$-product [19] and the author's $B$-product [3] as multiplicative binary operation.

We conclude our discussion with some examples.

As mentioned earlier, Narkiewicz in his paper [19] defined $A$-product as follows: For every positive integer $n$ fix the set $A_{n}$ of some divisors of $n$. For arithmetic functions $f$ and $g$ their $A$-product $f *_{A} g$ is given by:

$$
\left(f *_{A} g\right)(n)=\sum_{d \in A_{n}} f(d) g\left(\frac{n}{d}\right)
$$

for $n=1,2,3, \ldots$. 
The author in his paper [3] defined $B$-product as follows: For every positive integer $n$, fix the set $B_{n}$ of some pairs of divisors of $n$. For arithmetic functions $f$ and $g$ their $B$-product $f *_{B} g$ is given by:

$$
\left(f *_{B} g\right)(n)=\sum_{(u, v) \in B_{n}} f(u) g(v)
$$

for $n=1,2,3, \ldots$.

This $B$-product generalizes simultaneously the $A$-product of Narkiewicz [19] and the L.C.M. product and it has a non-void intersection with the $\Psi$-product of Lehmer [17]. The $\tau$-product of Scheid [20] is also a particular case of $B$-product.

Narkiewicz in his paper [19] called an $A$-product to be multiplicative if and only if $A_{m n}=A_{m} \times A_{n}$ for every pair $(m, n)$ of relatively prime positive integers (here $B \times C$ denotes the set of all integers, which can be represented in the form $b c, b \in B, c \in C)$.

The present author in his paper [4] defined a $B$-product to be multiplicative if and only if $B_{m n}=\left\{\left(r_{1} r_{2}, s_{1} s_{2}\right) \mid\left(r_{1}, s_{1}\right) \in B_{m},\left(r_{2}, \mathrm{~s}_{2}\right) \in B_{n}\right\}$ for every pair $(m, n)$ of relatively prime positive integers; in other words a $B$-product is multiplicative if and only if the following conditions hold: For every pair $(m, n)$ of relatively prime positive integers we have $(r, s) \in B_{m n}$ if and only if $\left(r^{(m)}, s^{(m)}\right) \in B_{m}$ and $\left(r^{(n)}, s^{(n)}\right) \in B_{n}$, where $r^{(k)}$ stands for the greatest common divisor of $r$ and $k$.

In the next section, we shall study in details some properties of $\boldsymbol{G M}$-functions and $\boldsymbol{G A}$-functions using Narkiewicz's $A$-product and the author's $B$-product as multiplicative binary operations.

\section{Main results}

\subsection{GM-Function and its properties}

As mentioned in our discussion earlier, Zafrullah in his paper [26] introduced a new generalization of multiplicative functions where he defined an arithmetic function $F$ to be a generalized multiplicative function (or $\boldsymbol{G M}$-function) if $F(1)=1$ and there exists a multiplicative function $f$ such that $F(m n)=F(m)^{f(n)} F(n)^{f(m)}$, whenever $(m, n)=1$. We now prove some results related to $\boldsymbol{G M}$-functions using Narkeiwicz's $A$-product and the author's $B$-product as the multiplicative binary operations.

Theorem 2.1.1. Let $F$ be a $\boldsymbol{G M}$-function and $h$ be a multiplicative function and let the $A$-product be multiplicative, then the arithmetic function $H$ defined by

$$
H(n)=\prod_{d \in A_{n}} F(d)^{h\left(\frac{n}{d}\right)}
$$

for all $n \in \mathbb{N}$ is a $\boldsymbol{G M}$-function.

Proof: We have

$$
H(1)=\prod_{d \in A_{1}} F(d)^{h\left(\frac{n}{d}\right)}=F(1)^{h\left(\frac{1}{1}\right)}=F(1)=1
$$

Let $(m, n)=1$, then 


$$
\begin{gathered}
H(m n)=\prod_{d \in \mathrm{A}_{m n}} F(d)^{h\left(\frac{m n}{d}\right)} \\
=\prod_{d_{1} \in \mathrm{A}_{m}, d_{2} \in \mathrm{A}_{n}} F\left(d_{1} d_{2}\right)^{h\left(\frac{m n}{d_{1} d_{2}}\right)} \\
=\prod_{d_{1} \in \mathrm{A}_{m}} \prod_{d_{2} \in \mathrm{A}_{n}}\left[F\left(d_{1}\right)^{f\left(d_{2}\right)} F\left(d_{2}\right)^{f\left(d_{1}\right)}\right]
\end{gathered}
$$

(where $f$ is a multiplicative function)

$$
\begin{gathered}
=\left[\prod _ { d _ { 1 } \in A _ { m } } F ( d _ { 1 } ) ^ { h ( \frac { m } { d _ { 1 } } ) ] ^ { \sum _ { d _ { 2 } \in \mathrm { A } _ { n } } } f ( d _ { 2 } ) h ( \frac { n } { d _ { 2 } } ) } \left[\prod_{d_{2} \in \mathrm{A}_{n}} F\left(d_{2}\right)^{\left.h\left(\frac{n}{d_{2}}\right)\right]^{\sum_{d_{1} \in \mathrm{A}_{m}}} f\left(d_{1}\right) h\left(\frac{m}{d_{1}}\right)}\right.\right. \\
=\left[\prod_{d_{1} \in \mathrm{A}_{m}} F\left(d_{1}\right)^{h\left(\frac{m}{d_{1}}\right)}\right]^{\left(f *_{A} h\right)(n)}\left[\prod_{d_{2} \in \mathrm{A}_{n}} F\left(d_{2}\right)^{h\left(\frac{n}{d_{2}}\right)}\right]^{\left(f *_{A} h\right)(m)} \\
=H(m)^{v(n)} \times H(n)^{v(m)},
\end{gathered}
$$

where $v=f *_{A} h$.

Since $f$ and $h$ are multiplicative functions and the $A$-product is multiplicative, therefore $v$ is a multiplicative function.

Thus, $H$ is a $\boldsymbol{G M}$-function.

Theorem 2.1.2. Let $F$ be a $\boldsymbol{G M}$-function and $h_{1}$ and $h_{2}$ be multiplicative functions and let the $A$-product be multiplicative, then the arithmetic function $H$ defined by

$$
H(n)=\prod_{d \in A_{n}} F(d)^{h_{1}(d) h_{2}\left(\frac{n}{d}\right)}
$$

for all $n \in \mathbb{N}$ is a $\boldsymbol{G M}$-function.

Proof: Let $F(d)^{h_{1}(d)}=K(d)$. Then

$$
H(n)=\prod_{d \in A_{n}} K(d)^{h_{2}\left(\frac{n}{d}\right)}
$$

and $K(1)=F(1)^{h_{1}(1)}=1$. Let $(m, n)=1$, then

$$
\begin{gathered}
K(m n)=F(m n)^{h_{1}(m n)} \\
=\left[F(m)^{f(n)} F(n)^{f(m)}\right]^{h_{1}(m) h_{1}(n)}
\end{gathered}
$$

(where $f$ is a multiplicative function)

$$
\begin{aligned}
& =K(m)^{f h_{1}(n)} K(n)^{f h_{1}(m)} \\
& =K(m)^{v(n)} K(n)^{v(m)}
\end{aligned}
$$

Since $f$ and $h_{1}$ are multiplicative functions, then $v=f h_{1}$ is also a multiplicative function. Therefore, $K$ is a $\boldsymbol{G M}$-function.

Therefore, by Theorem 2.1.1 we conclude that $H$ is a $\boldsymbol{G M}$-function. 
Theorem 2.1.3. Let $F$ be a $\boldsymbol{G M}$-function and $h$ be a multiplicative function and let the $B$-product be multiplicative. Then the arithmetic function $J(n)$ given by

$$
J(n)=\prod_{(r, s) \in B_{n}} F(r)^{h(s)}
$$

for all $n \in \mathbb{N}$ is a $\boldsymbol{G M}$-function.

Proof: We have

$$
J(1)=\prod_{(r, s) \in B_{1}} F(r)^{h(s)}=F(1)^{h(1)}=1
$$

Let $(m, n)=1$. Then

$$
\begin{aligned}
& J(m n)=\prod_{(r, s) \in B_{m n}} F(r)^{h(s)} \\
& =\prod_{\left(r^{(m)}, s^{(m)}\right) \in B_{m}} \prod_{\left(r^{(n)}, s^{(n)}\right) \in B_{n}}^{(r, s) \in B_{m n}} F\left(r^{(m)} r^{(n)}\right)^{h\left(s^{(m)} s^{(n)}\right)} \\
& =\prod_{\left(r^{(m)}, s^{(m)}\right) \in B_{m}} \prod_{\left(r^{\left.(n), s^{(n)}\right) \in B_{n}}\right.}\left[F\left(r^{(m)}\right)^{f\left(r^{(n)}\right)} F\left(r^{(n)}\right)^{f\left(r^{(m)}\right)}\right]^{h\left(s^{(m)}\right) h\left(s^{(n)}\right)}
\end{aligned}
$$

(where $f$ is a multiplicative function)

$$
\begin{aligned}
& =\quad \prod_{\left(r^{(m), S^{(m)}}\right) \in B_{m}} \prod_{\left(r^{\left.(n), S^{(n)}\right) \in B_{n}}\right.}\left[F\left(r^{(m)}\right)^{h\left(s^{(m)}\right)}\right]^{f\left(r^{(n)}\right) h\left(s^{(n)}\right)} \times\left[F\left(r^{(n)}\right)^{h\left(s^{(n)}\right)}\right]^{f\left(r^{(m)}\right) h\left(s^{(m)}\right)} \\
& =\left[\left(\prod_{\left(r^{(m)}, s^{(m)}\right) \in B_{m}}\left[F\left(r^{(m)}\right)^{h\left(s^{(m)}\right)}\right]\right)^{\sum_{\left(r^{(n), s}(n)\right) \in B_{n}} f\left(r^{(n)}\right) h\left(s^{(n)}\right)}\right] \\
& \times\left[\left(\prod_{\left(r^{(n), S}(n)\right) \in B_{n}}\left[F\left(r^{(n)}\right)^{h\left(s^{(n)}\right)}\right]\right)^{\sum_{\left(r^{(m), s}(m)\right) \in B m} f\left(r^{(m)}\right) h\left(s^{(m)}\right)}\right] \\
& =J(m)^{\left(f *_{B} h\right)(n)} \times J(n)^{\left(f *_{B} h\right)(m)} \\
& =J(m)^{v(n)} \times J(n)^{v(m)}
\end{aligned}
$$

where $v=f *_{B} h$.

Since $f$ and $h$ are multiplicative functions and the $B$-product is multiplicative, therefore $v$ is a multiplicative function.

Hence, $J$ is a $\boldsymbol{G M}$-function.

Theorem 2.1.4. Let $F$ be a $\boldsymbol{G M}$-function and let $h_{1}$ and $h_{2}$ be multiplicative functions and let the $B$-product be multiplicative. Then the arithmetic function $J$ defined by

$$
J(n)=\prod_{(r, s) \in B_{n}} F(r)^{h_{1}(r) h_{2}(s)}
$$

for all $n \in \mathbb{N}$ is a $\boldsymbol{G M}$-function.

Proof: Let $U(r)=F(r)^{h_{1}(r)}$. Therefore, 


$$
J(n)=\prod_{(r, s) \in B_{n}} U(r)^{h_{2}(s)}
$$

Clearly,

$$
U(1)=F(1)^{h_{1}(1)}=1
$$

Let $(m, n)=1$.Then

$$
U(m n)=F(m n)^{h_{1}(m n)}=\left(F(m)^{f(n)} F(n)^{f(m)}\right)^{h_{1}(m) h_{1}(n)}
$$

(where $f$ is a multiplicative function)

$$
\begin{gathered}
=\left[F(m)^{f(n)}\right]^{h_{1}(m) h_{1}(n)}\left[F(n)^{f(m)}\right]^{h_{1}(m) h_{1}(n)} \\
=\left[F(m)^{h_{1}(m)}\right]^{f(n) h_{1}(n)}\left[F(n)^{h_{1}(n)}\right]^{f(m) h_{1}(m)} \\
=U(m)^{v(n)} U(n)^{v(m)}
\end{gathered}
$$

where $v=f h_{1}$, is a multiplicative function.

Therefore, $U$ is a $\boldsymbol{G M}$-function.

Hence, by Theorem 2.1.3 we conclude that $J$ is a $\boldsymbol{G M}$-function.

\section{$2.2 G A$-Function and its properties}

An arithmetic function $F$ is said to be additive if $F(m n)=F(m)+F(n)$ whenever $(m, n)=1$. We define an arithmetic function $F$ to be a generalized additive function (or briefly a $\boldsymbol{G A}$-function) if there exists a multiplicative function $f$ such that $F(m n)=F(m) f(n)+F(n) f(m)$ whenever $(m, n)=1$. Chawla [9] calls such a function a distributive function. One can notice that the notion of $\boldsymbol{G A}$-function is an additive analogue of $\boldsymbol{G} \boldsymbol{M}$-function. We now prove some results on $\boldsymbol{G A}$-function in the context of Narkiewicz's $A$-product and the author's $B$-product.

Theorem 2.2.1. Let $F$ be a $\boldsymbol{G A}$-function and $h$ be a multiplicative function and let the $A$-product be multiplicative. Then the arithmetic function $H$ defined by

$$
H(n)=\sum_{d \in A_{n}} F(d) h(d)
$$

for all $n \in \mathbb{N}$, is a $\boldsymbol{G A}$-function.

Proof: Let $U(d)=F(d) h(d)$ and $(m, n)=1$. Then

$$
\begin{gathered}
U(m n)=F(m n) h(m n) \\
=[F(m) f(n)+F(n) f(m)] h(m) h(n)
\end{gathered}
$$

(where $f$ is a multiplicative function)

$$
\begin{gathered}
=F(m) h(m) f(n) h(n)+F(n) h(n) f(m) h(m) \\
=U(m) v(n)+U(n) v(m)
\end{gathered}
$$

(where $v=f h$ is a multiplicative function).

Therefore, $U$ is a $\boldsymbol{G A}$-function. Now 


$$
H(m n)=\sum_{d \in A_{m n}} U(d)=\sum_{d_{1} \in A_{m}} \sum_{d_{2} \in A_{n}} U\left(d_{1} d_{2}\right)
$$

As $d_{1} \in A_{m}, d_{2} \in A_{n}$ so $\left(d_{1}, d_{2}\right)=1$, and since $U$ is a $\boldsymbol{G A}$-function, therefore there exists a multiplicative function $f$ such that $U\left(d_{1} d_{2}\right)=U\left(d_{1}\right) f\left(d_{2}\right)+U\left(d_{2}\right) f\left(d_{1}\right)$. Therefore,

$$
\begin{gathered}
H(m n)=\sum_{d_{1} \in A_{m}} \sum_{d_{2} \in A_{n}}\left[U\left(d_{1}\right) f\left(d_{2}\right)+U\left(d_{2}\right) f\left(d_{1}\right)\right] \\
=\sum_{d_{1} \in A_{m}} \sum_{d_{2} \in A_{n}} U\left(d_{1}\right) f\left(d_{2}\right)+\sum_{d_{1} \in A_{m}} \sum_{d_{2} \in A_{n}} U\left(d_{2}\right) f\left(d_{1}\right) \\
=H(m) w(n)+H(n) w(m),
\end{gathered}
$$

where

$$
w(m)=\sum_{d_{1} \in A_{m}} f\left(d_{1}\right), w(n)=\sum_{d_{2} \in A_{n}} f\left(d_{2}\right)
$$

are multiplicative functions.

Hence, $H$ is a $\boldsymbol{G A}$-function.

Theorem 2.2.2. Let $F$ be a $\boldsymbol{G A}$-function and $h_{1}$ and $h_{2}$ be multiplicative functions and let the $A$-product be multiplicative. Then the arithmetic function $H$ defined by

$$
H(n)=\sum_{d \in A_{n}} F(d) h_{1}(d) h_{2}\left(\frac{n}{d}\right)
$$

for all $n \in \mathbb{N}$, is a $\boldsymbol{G A}$-function.

Proof: Let $U(d)=F(d) h_{1}(d)$. Then from the proof of Theorem 2.2.1, $U$ is a $\boldsymbol{G A}$-function. Now

$$
H(n)=\sum_{d \in A_{n}} U(d) h_{2}\left(\frac{n}{d}\right)
$$

Let $(m, n)=1$. Then

$$
\begin{aligned}
& H(m n)=\sum_{d \in A_{m n}} U(d) h_{2}\left(\frac{m n}{d}\right) \\
= & \sum_{d_{1} \in A_{m}} \sum_{d_{2} \in A_{n}} U\left(d_{1} d_{2}\right) h_{2}\left(\frac{m n}{d_{1} d_{2}}\right)
\end{aligned}
$$

As $d_{1} \in A_{m}, d_{2} \in A_{n}$ so $\left(d_{1}, d_{2}\right)=1$, and since $U$ is a $\boldsymbol{G A}$-function, therefore there exists a multiplicative function $f$ such that

Therefore,

$$
U\left(d_{1} d_{2}\right)=U\left(d_{1}\right) f\left(d_{2}\right)+U\left(d_{2}\right) f\left(d_{1}\right) .
$$

$$
\begin{aligned}
& H(m n)=\sum_{d_{1} \in A_{m}} \sum_{d_{2} \in A_{n}}\left[U\left(d_{1}\right) f\left(d_{2}\right)+U\left(d_{2}\right) f\left(d_{1}\right)\right] \times\left[h_{2}\left(\frac{m}{d_{1}}\right) h_{2}\left(\frac{n}{d_{2}}\right)\right] \\
= & \sum_{d_{1} \in A_{m}} \sum_{d_{2} \in A_{n}}\left[U\left(d_{1}\right) f\left(d_{2}\right) h_{2}\left(\frac{m}{d_{1}}\right) h_{2}\left(\frac{n}{d_{2}}\right)+U\left(d_{2}\right) f\left(d_{1}\right) h_{2}\left(\frac{m}{d_{1}}\right) h_{2}\left(\frac{n}{d_{2}}\right)\right] \\
= & \sum_{d_{1} \in A_{m}} \sum_{d_{2} \in A_{n}} U\left(d_{1}\right) h_{2}\left(\frac{m}{d_{1}}\right) f\left(d_{2}\right) h_{2}\left(\frac{n}{d_{2}}\right)+\sum_{d_{1} \in A_{m}} \sum_{d_{2} \in A_{n}} U\left(d_{2}\right) h_{2}\left(\frac{n}{d_{2}}\right) f\left(d_{1}\right) h_{2}\left(\frac{m}{d_{1}}\right)
\end{aligned}
$$




$$
\begin{gathered}
=\sum_{d_{1} \in A_{m}} U\left(d_{1}\right) h_{2}\left(\frac{m}{d_{1}}\right) \sum_{d_{2} \in A_{n}} f\left(d_{2}\right) h_{2}\left(\frac{n}{d_{2}}\right)+\sum_{d_{2} \in A_{n}} U\left(d_{2}\right) h_{2}\left(\frac{n}{d_{2}}\right) \sum_{d_{1} \in A_{m}} f\left(d_{1}\right) h_{2}\left(\frac{m}{d_{1}}\right) \\
=\left[\left(U *_{A} h_{2}\right)(m)\right] .\left[\left(f *_{A} h_{2}\right)(n)\right]+\left[\left(U *_{A} h_{2}\right)(n)\right] .\left[\left(f *_{A} h_{2}\right)(n)\right] \\
=H(m) \cdot v(n)+H(n) \cdot v(m)
\end{gathered}
$$

where $v(k)=\left(f *_{A} h_{2}\right)(k)$.

Now since $f$ and $h_{2}$ are multiplicative functions and the $A$-product is multiplicative, therefore $v=f *_{A} h_{2}$ is a multiplicative function. Consequently $H$ is a $\boldsymbol{G A}$-function.

Theorem 2.2.3. Let $F$ be a $\boldsymbol{G A}$-function and let $h_{1}$ and $h_{2}$ be multiplicative functions and let the $B$-product be multiplicative, then the arithmetic function $H$ defined by

$$
H(n)=\sum_{(r, s) \in B_{n}} F(r) h_{1}(r) h_{2}(s)
$$

for all $n \in \mathbb{N}$ is a $\boldsymbol{G A}$-function.

Proof: Let $U(d)=F(d) h_{1}(d)$. Then, from the proof of Theorem 2.2.1, $U$ is a $\boldsymbol{G A}$-function. Therefore,

$$
H(n)=\sum_{(r, s) \in B_{n}} U(r) h_{2}(s)
$$

Let $(m, n)=1$.Then

$$
\begin{gathered}
H(m n)=\sum_{(r, s) \in B_{m n}} U(r) h_{2}(s) \\
=\sum_{\left(r^{(m)}, s^{(m)}\right) \in B_{m}} \sum_{\left(r^{(n)}, s^{(n)}\right) \in B_{n}} U\left(r^{(m)} r^{(n)}\right) h_{2}\left(s^{(m)} s^{(n)}\right)
\end{gathered}
$$

As $\left(r^{(m)}, s^{(m)}\right) \in B_{m}$ and $\left(r^{(n)}, s^{(n)}\right) \in B_{n}$, so $\left(r^{(m)}, r^{(n)}\right)=1$ and $\left(s^{(m)}, s^{(n)}\right)=1$ and since $U$ is a $\boldsymbol{G A}$-function, therefore, there exists a multiplicative function $f$ such that

$$
U\left(r^{(m)} r^{(n)}\right)=U\left(r^{(m)}\right) f\left(r^{(n)}\right)+U\left(r^{(n)}\right) f\left(r^{(m)}\right)
$$

Therefore

$$
\begin{aligned}
& H(m n)=\sum_{\left(r^{(m)}, s^{(m)}\right) \in B_{m}} \sum_{\left(r^{\left.(n), s^{(n)}\right) \in B_{n}}\right.}\left[U\left(r^{(m)}\right) f\left(r^{(n)}\right)+U\left(r^{(n)}\right) f\left(r^{(m)}\right)\right]\left[h_{2}\left(s^{(m)} s^{(n)}\right)\right] \\
& =\sum_{\left(r^{(m)}, s^{(m)}\right) \in B_{m}} \sum_{\left(r^{(n)}, s^{(n)}\right) \in B_{n}}\left[U\left(r^{(m)}\right) f\left(r^{(n)}\right) h_{2}\left(s^{(m)}\right) h_{2}\left(s^{(n)}\right)\right. \\
& \left.+U\left(r^{(n)}\right) f\left(r^{(m)}\right) h_{2}\left(s^{(m)}\right) h_{2}\left(s^{(n)}\right)\right] \\
& \left.=\sum_{\left(r^{(m)}, s^{(m)}\right) \in B_{m}} \sum_{\left(r^{\left.(n), s^{(n)}\right) \in B_{n}}\right.} U\left(r^{(m)}\right) f\left(r^{(n)}\right) h_{2}\left(s^{(m)}\right) h_{2}\left(s^{(n)}\right)\right) \\
& \left.+\sum_{\left(r^{(m)}, s^{(m)}\right) \in B_{m}} \sum_{\left(r^{(n)}, s^{(n)}\right) \in B_{n}} U\left(r^{(n)}\right) f\left(r^{(m)}\right) h_{2}\left(s^{(m)}\right) h_{2}\left(s^{(n)}\right)\right)
\end{aligned}
$$




$$
\begin{gathered}
=\sum_{\left(r^{(m)}, s^{(m)}\right) \in B_{m}} U\left(r^{(m)}\right) h_{2}\left(s^{m}\right) \sum_{\left(r^{(n)}, s^{(n)}\right) \in B_{n}} f\left(r^{(n)} h_{2}\left(s^{(n)}\right)\right) \\
+\sum_{\left(r^{\left.(n), s^{(n)}\right) \in B_{n}}\right.} U\left(r^{(n)}\right) h_{2}\left(s^{(n)}\right) \sum_{\left(r^{(m)}, s^{(m)}\right) \in B_{m}} f\left(r^{(m)} h_{2}\left(s^{(m)}\right)\right) \\
=H(m)\left(f *_{B} h_{2}\right)(n)+H(n)\left(f *_{B} h_{2}\right)(m) .
\end{gathered}
$$

Now since $f$ and $h_{2}$ are multiplicative functions and the $B$-product is multiplicative, therefore, $f *_{B} h_{2}$ is a multiplicative function. Consequently, $H$ is a $\boldsymbol{G A}$-function.

We conclude our discussion with some examples.

\section{Examples}

Example 1. If

$$
H(n)=\prod_{d \in A_{n}} d^{d}
$$

for all $n \in \mathbb{N}$, and the $A$-product is multiplicative, then $H$ is a $\boldsymbol{G M}$-function.

Proof. We have

$$
H(n)=\prod_{d \in A_{n}} d^{d}
$$

Let $F(d)=d^{d}$, then for every relatively prime positive integers $m, n$ we have

$$
F(m n)=(m n)^{m n}=m^{m n} n^{m n}=\left(m^{m}\right)^{n}\left(n^{n}\right)^{m}=F(m)^{n} F(n)^{m}=F(m)^{f(n)} F(n)^{f(m),}
$$

Where $f(u)=u$ for every $u \in \mathbb{N}$, is a multiplicative function. Therefore, $F$ is a $\boldsymbol{G M}$-function. Now

$$
H(m n)=\prod_{d \in A_{m n}} F(d)=\prod_{d_{1} d_{2} \in A_{m n}} F\left(d_{1} d_{2}\right),
$$

(where $d=d_{1} d_{2}, d_{1} \mid m$ and $d_{2} \mid n$ )

$$
=\prod_{d_{1} d_{2} \in A_{m n}} F\left(d_{1}\right)^{g\left(d_{2}\right)} F\left(d_{2}\right)^{g\left(d_{1}\right)}
$$

(where $g$ is a multiplicative function)

$$
\begin{gathered}
=\left\{\left[\prod_{d_{1} \in A_{m}} F\left(d_{1}\right)\right]^{\sum_{d_{2} \in A_{n}} g\left(d_{2}\right)}\right\} \times\left\{\left[\prod_{d_{2} \in A_{n}} F\left(d_{2}\right)\right]^{\sum_{d_{1} \in A_{m}} g\left(d_{1}\right)}\right\} \\
=H(m)^{u(n)} \times H(n)^{u(m)}
\end{gathered}
$$

where $u(m)=\sum_{d_{1} \in A_{m}} g\left(d_{1}\right)$ and $u(n)=\sum_{d_{2} \in A_{n}} g\left(d_{2}\right)$

Since $g$ is a multiplicative function and the $A$-product is multiplicative, therefore, $u$ is a multiplicative function. Hence, $H$ is a $\boldsymbol{G} \boldsymbol{M}$-function.

Example 2. If

$$
H(n)=\prod_{(r, s) \in B_{n}} r^{s}
$$


for all $n \in \mathbb{N}$, and the $B$-product is multiplicative, then $H$ is a $\boldsymbol{G M}$-function.

Proof. Let $F(r)=r$ and $h(s)=s$, then $F(m n)=m n=m^{\eta(n)} n^{\eta(m)}=F(m)^{\eta(n)} F(n)^{\eta(m)}$, where $\eta(k)=1$ for every $k \in \mathbb{N}$ is a multiplicative function and therefore $F$ is a $\boldsymbol{G M}$-function. Now since $h(s)=s$ is a multiplicative function, and the $B$-product is multiplicative, therefore by Theorem 2.1.3

is a $\boldsymbol{G M}$-function.

$$
H(n)=\prod_{(r, s) \in B_{n}} r^{s}=\prod_{(r, s) \in B_{n}} F(r)^{h(s)}
$$

\section{Example 3. If}

$$
H(n)=\sum_{d \in A_{n}} d \log d
$$

for all $n \in \mathbb{N}$, and the $A$-product is multiplicative, then $H$ is a $\boldsymbol{G A}$-function.

Proof. Let $(m, n)=1$, then

$$
\begin{gathered}
H(m n)=\sum_{d \in A_{m} n} d \log d \\
=\sum_{d_{1} \in A_{m}, d_{2} \in A_{n}}\left(d_{1} d_{2}\right) \log \left(d_{1} d_{2}\right) \\
=\sum_{d_{1} \in A_{m}, d_{2} \in A_{n}}\left(d_{1} d_{2}\right)\left[\log d_{1}+\log d_{2}\right] \\
=\sum_{d_{1} \in A_{m}, d_{2} \in A_{n}}\left(d_{1} \log d_{1}\right) d_{2}+\sum_{d_{1} \in A_{m}}\left(d_{2} \log d_{2}\right) d_{1} \\
=d_{1} \log d_{1} \sum_{d_{2} \in A_{n}, d_{2} \in A_{n}} d_{2}+\sum_{d_{2} \in A_{n}} d_{2} \log d_{2} \sum_{d_{1} \in A_{m}} d_{1} \\
=H(m) \sum_{d_{2} \in A_{n}} d_{2}+H(n) \sum_{d_{1} \in A_{m}} d_{1}
\end{gathered}
$$

where

$$
\alpha(k)=\sum_{d \in A_{k}} d=\sum_{d \in A_{k}} h(d)
$$

and $h(s)=s$ for every $s \in \mathbb{N}$. Since $h$ is a multiplicative function and the $A$-product is multiplicative, therefore $\alpha$ is a multiplicative function. Hence $H$ is a $\boldsymbol{G A}$-function.

\section{Example 4. If}

$$
H(n)=\sum_{(r, s) \in B_{n}} r \log s
$$

for all $n \in \mathbb{N}$, and the $B$-product is multiplicative, then $H$ is a $\boldsymbol{G A}$-function.

Proof. Let $(m, n)=1$. Then

$$
H(m n)=\sum_{(r, s) \in B_{m n}} r \log s
$$




$$
\begin{gathered}
=\sum_{\left(r^{(m)}, s^{(m)}\right) \in B_{m},\left(r^{(n)}, s^{(n)}\right) \in B_{n}} r^{(m)} r^{(n)} \log \left(s^{(m)} s^{(n)}\right) \\
=\sum_{\left(r^{(m)}, s^{(m)}\right) \in B_{m},\left(r^{(n)}, s^{(n)}\right) \in B_{n}} r^{(n)}\left[\log \left(s^{(m)}\right)+\log \left(s^{(n)}\right)\right] \\
=\sum_{\left(r^{(m)}, s^{(m)}\right) \in B_{m}} \sum_{r^{(m)} \log \left(s^{(m)}\right) r^{(n)}+\sum_{\left(r^{(m)}, s^{(n)}\right) \in B_{n}} r_{\left(r^{(m)}\right) \in B_{m},\left(r^{(n)}, s^{(n)}\right) \in B_{n}} r^{(n)} \log \left(s^{(n)}\right) r^{(m)} \sum_{\left(r^{(n)}, s^{(n)}\right) \in B_{n}} r^{(n)} \log \left(s^{(n)}\right)} \sum_{\left(r^{(m)}, s^{(m)}\right) \in B_{m}} r^{(m)} \\
=H(m) \alpha(n)+H(n) \alpha(m)
\end{gathered}
$$

where

and $h(s)=s$ for every $s \in \mathbb{N}$.

$$
\alpha(k)=\sum_{\left(r^{(k)}, s^{(k)}\right) \in B_{k}} r^{(k)}=\sum_{\left(r^{(k)}, s^{(k)}\right) \in B_{k}} h\left(r^{(k)}\right),
$$

Since $h$ is a multiplicative function and the $B$-product is multiplicative, therefore $\alpha$ is a multiplicative function. Hence $H$ is a $\boldsymbol{G A}$-function.

\section{References}

[1] Apostol, T. M. (1976). Introduction to Analytic Number Theory, Springer-Verlag, New York.

[2] Bell, E. T. (1915). Arithmetical Theory of Certain Numerical Functions. Volume 1, University of Washington.

[3] Bhattacharjee, D. (1997). B-product and its properties. Bulletin of Pure and Applied Sciences, 16(1), 77-83.

[4] Bhattacharjee, D. (1998). Multiplicative B-product and its properties. Georgian Mathematical Journal, 5(4), 315-320.

[5] Bhattacharjee, D. (2002). A Generalized B-product and its properties, Bulletin of the Allahabad Mathematical Society, 17, 17-21.

[6] Bhattacharjee, D. (2005). Multiplicative $K_{B}$-product and its properties.Bulletin of the Calcutta Mathematical Society, 97(2), 153-162.

[7] Bhattacharjee, D. (2007). On Some New Arithmetical Convolutions. Proceedings of the 2007 International Conference on High Performance Computing Networking and Communication Systems (HPCNCS-07), 9-12 July 2007, Orlando, FL, USA, 26-30.

[8] Bhattacharjee, D., \& Saikia, P. K. (2012). On Some New Class of Arithmetic Convolutions Involving Arbitrary Sets of Integers. Romanian Journal of Mathematics and Computer Science, 2(2), 23-35.

[9] Chawla, L. M. (1973). Distributive arithmetical functions of two or more variables. Journal of Natural Sciences Math., 13, 263-270. 
[10] Pellegrino, F. (1953) Sviluppi moderni del Calcolo numerico integrale di Michele Cipolla. IV Congresso dell' Unione Matematica Italiana-Taormina, 2, 161-168.

[11] Cohen, E. (1959).Arithmetical functions associated with arbitrary sets of integers, Acta Arithmetica, 5, 407-415.

[12] Davison, T. M. K. (1966). On Arithmetic Convolutions. Canadian Mathematical Bulletin, 9, 287-296.

[13] Dickson, L. E.(1919).History of the Theory of Numbers, Washington: Carnegie Institution.

[14] Gioia, A. A. (1965).The $K$-product of the arithmetic functions, Canadian Journal of Mathematics, 17, 970-976.

[15] Haukkanen, P. (1992). A note on generalized multiplicative and generalized additive arithmetic functions. The Mathematics Student, 61(1-4), 113-116.

[16] Haukkanen, P. (1996). On a Binomial Convolution of Arithmetic Functions. Nieuw Archief voor Wiskunde, 14(2), 209-216.

[17] Lehmer, D. H. (1931). A New Calculus of Numerical Functions. American Journal of Mathematics, 53, 843-854.

[18] McCarthy, P. J. (1986). Introduction to Arithmetical Functions, Springer-Verlag, New York.

[19] Narkiewicz, W. (1963). On a class of arithmetical convolutions. Colloquium Mathematicum, 10, 81-94.

[20] Scheid, H. (1969). Einige Ringe zahlentheoretischer Funktionen. Journal für die reine und angewandte Mathematik, 1969(237), 1-11.

[21] Sivaramakrishnan, R. (1989). Classical Theory of Arithmetical Functions, Monographs and Textbooks in Pure and Applied Mathematics, 126, Marcel-Dekker Inc., New York.

[22] Subbarao, M. V. (1972). On Some Arithmetical Convolutions, Lecture Notes in Mathematics, Springer, 251, 247-271.

[23] Tóth, L. (2002). On a Class of Arithmetic Convolutions involving Arbitrary Sets of Integers. Mathematica Pannonica, 13, 249-263.

[24] Tóth, L. (2004). On a Certain Arithmetic Functions involving Exponential Divisors. Annales Univ. Sci. Budapest., Sect. Comp., 24, 285-294.

[25] Vaidyanathaswamy, R. (1931). The Theory of Multiplicative Arithmetical Functions. Transactions of the American Mathematical Society, 33, 579-662.

[26] Zafrullah, M. (1988). On generalized multiplicative functions. Journal of Natural Sciences and Mathematics, 28, 257-268. 